\title{
Cuento y géneros próximos en revistas literarias colombianas (1900- 1951): un balance ${ }^{1}$
}

\author{
Ana María Agudelo Ochoa ${ }^{2}$
}

Resumen. A partir del estudio de 24 revistas literarias colombianas, se analizan algunos aspectos relativos a las dinámicas del cuento en el país. Se reflexiona, principalmente, acerca de la noción de cuento subyacente en las publicaciones, el lugar de las narraciones en la sintaxis de las revistas, la presencia tanto de autores colombianos como foráneos. Asimismo se enuncian algunos problemas y fenómenos que merecen revisión detallada. Todo lo anterior con el objetivo de aportar al estudio histórico del cuento en Colombia.

Palabras clave: cuento; publicaciones periódicas, siglo XX; historia de la literatura colombiana.

\section{[en] Stories and upcoming genres in Colombian literary magazines (1900- 1951): a balance}

\begin{abstract}
The aim of this paper is to analyzed some aspects related to the dynamics of the short story in Colombia. To achieve this objective 24 Colombian literary magazines were studied. It is mainly reflected on the notion of underlying short story in the publications, the place of the narratives in the syntax of the magazines, the presence of both Colombian and foreign authors. It also lists some problems and phenomena that deserve detailed review. All with the aim of contributing to the historical study of the story in Colombia.
\end{abstract}

Keywords: Short Story; Periodicals from the 20th Century; History of Colombian Literature.

Sumario. 1. Introducción. 2. Cuento: uso del concepto y lugar del género en la sintaxis de las revistas. 2.1. Un género de difícil definición. 2.2. Hacia una noción de cuento en las revistas. 2.3. El lugar del texto en la revista: hacia la especialización del medio. 3. El cuento en las revistas: los datos hablan. 3.1. Publicación de cuento en las revistas. 3.2. El cuento: autores y procedencia. 4. Proyecciones de la investigación. A manera de conclusión.

Cómo citar: Agudelo Ochoa, A. (2018) Cuento y géneros próximos en revistas literarias colombianas (1900-1951): un balance, en Anales de Literatura Hispanoamericana 47, 17-36.

1 Artículo derivado de la investigación "El cuento colombiano en las revistas literarias colombianas (19001950). Estudio histórico y hemerográfico”, asimismo se inscribe en la Estrategia de Sostenibilidad para grupos de investigación Universidad de Antioquia, UdeA, 2016-2017.

2 Universidad de Antioquia, Medellín. Colombia.

E-mail: amaria.agudelo@udea.edu.co 


\section{Introducción}

Este artículo expone un balance general de los hallazgos obtenidos a partir de la revisión de un corpus de 24 revistas literarias colombianas que otorgaron especial valor al cuento. ${ }^{3}$ Como indicamos en el texto que introduce esta sección monográfica, el examen de dicho corpus pretende aportar al estudio histórico de este género narrativo breve. Es preciso indicar que se propone una revisión transversal del corpus de revistas y por lo mismo debemos dejar de lado las singularidades del género en cada una de las publicaciones, terreno rico y complejo que exige estudios pormenorizados. La cantidad de datos, tablas y gráficos que expondremos puede resultar abrumadora, pero su inclusión obedece al interés por exponer información que sugiere fenómenos y plantea cuestiones que merecen estudios más concentrados en la singularidad. A lo largo del artículo enunciamos algunas hipótesis y preguntas para futuros proyectos. Nos ocuparemos, principalmente, del uso del término cuento por parte de las revistas y el lugar del género en la sintaxis de las mismas; posteriormente examinaremos la presencia de la cuentística, tanto colombiana como extranjera, y haremos algunos apuntes acerca de la relación entre sistemas literarios; por último, presentaremos algunas conclusiones que pretenden, sobre todo, proponer líneas de análisis. Cabe señalar que debido a la magnitud de los datos obtenidos y al carácter panorámico de este artículo, no es posible revisar en detalle todos los aspectos enunciados. En todo caso, algunos problemas y fenómenos puntuales serán abordados en los otros artículos que conforman este monográfico.

\section{Cuento: uso del concepto y lugar del género en la sintaxis de las revistas}

\subsection{Un género de difícil definición}

Debido a las dinámicas propias de los géneros literarios, los cuales no pueden ser tenidos por entidades estáticas, hemos optado por aludir tanto a "cuento" como a "géneros próximos", en aras de reconocer la hibridez y rasgos peculiares de ciertas manifestaciones literarias cercanas a esta forma narrativa. No desconocemos el carácter ambiguo, en ocasiones inclasificable, de los textos, característica que, por cierto, es una prueba de la complejidad del cuento y un factor de obligado análisis. La utilización del término "cuento" en las revistas revisadas — como parte del título del relato, de una sección e, incluso, de la publicación misma- fue un primer elemento para tener en cuenta por parte del equipo de investigadores durante el proceso de revisión de las fuentes e identificación de los textos. Sin embargo, no en todos los casos el impreso periódico acude a las etiquetas genéricas, de allí que se apele a la identificación de rasgos que consideramos propios del género a la hora de catalogar los textos: ficcionalidad, narratividad y brevedad (Pacheco 1993). ${ }^{4}$

3 Las singularidades del proceso de revisión y el dato de los investigadores que participaron en el mismo se indicaron en el texto que introduce la sección monográfica.

4 Acudimos a las consideraciones de Carlos Pacheco dado el amplio carácter de su propuesta, la cual permite mantener un criterio más abierto a la hora de clasificar los relatos detectados en las revistas revisadas. 
Asimismo las consideraciones de Marie-Eve Thérenty iluminaron la revisión de las fuentes en tanto permitieron considerar una poética del cuento determinada por una "matrice journalistique impérative" (2007). Esta historiadora afirma que la poética de algunos géneros literarios, entre los que se encuentra el cuento, está determinada por una suerte de esencia "periodística" que los constriñe debido a la profunda relación entre el texto y la materialidad que lo soporta. Así, al ser las publicaciones periódicas un espacio privilegiado del cuento durante el periodo que nos ocupa, una serie de rasgos propios de estas determinan los relatos. Thérenty señala entre esos rasgos: el efecto de sección, la periodicidad y la confluencia de múltiples voces (2007). ${ }^{5}$

\subsection{Hacia una noción de cuento en las revistas}

Si bien nuestras pesquisas cobijaron en general relatos que cumplieran una serie de rasgos que atribuimos al cuento, no podemos pasar por alto que el uso explícito del término "cuento" ofrece fuertes indicios acerca de la noción que del género tienen los autores y los editores. En el corpus revisado, la denominación "cuento" se atribuye a 1480 las entradas, como parte del título o subtítulo del relato, como parte del nombre de la sección donde la narración aparece publicada o como parte del título de la revista.

El término "cuento" aparece a lo largo de todo el periodo estudiado; algunas revistas que circularon durante la primera década del siglo XX lo utilizan como parte del título o subtítulo de alguna narración: "Cuentos breves. Cosas que mueren” (Max Grillo, La Gruta, 1902), "Cuentos cortos. Almas y flores”, "Cuentos cortos. Celos de muerto”, “Cuentos cortos. El leñateo” (Rafael Espinosa Guzmán [REG], La Gruta, 1903), "Los poemas de mi mujer. Cuento polaco" (Vicente Koriakuwictz, Revista Nueva, 1904), "El rey de la máscara (cuento color de sangre)" (Ramón del Valle Inclán, Lectura Amena, 1905). A lo largo de todo el periodo (1900-1951) aparecen narraciones tituladas con la palabra "cuento", en algunos casos con claro ánimo de clasificación, en otros como elemento estilístico del título ("Cuentas y cuentos”, Cromos, 1928, Alberto Sánchez; "Era un cuento, nada más”, Revista de las Indias, 1946, Antonio Cardona Jaramillo).

Con la sección "Cuentos nacionales", aparecida en 1910 en la revista El Gráfico, ubicamos el posible inicio del uso de un apartado exclusivo de cuento en las revistas colombianas. A esta sección seguirían otras en esta y demás revistas estudiadas; hemos logrado ubicar cerca de 86 secciones en el corpus revisado. Los nombres de las mismas sitúan al lector en un horizonte específico de género literario, además, en la mayoría de los casos, especifican aún más el carácter del texto mediante una marca geográfica, temporal, temática, estilística o alguna singularidad del autor (ver tabla 1). Algunas de las 86 secciones logran mantenerse

\footnotetext{
Las reflexiones teóricas de Therenty se enmarcan en un gran proyecto inscrito por sus gestores en la línea que ellos mismos denominan poética histórica, vertiente que se propone aportar al análisis histórico del acontecer literario sin descuidar ni la obra de arte literaria ni las particularidades del entorno socio-cultural de un sistema literario concreto. El propósito de la poética histórica es "estudiar de manera prioritaria la hechura literaria, la evolución de los modos de escritura, las limitaciones formales, los procesos de invención y renovación, y por lo tanto, de colocar en el primer plano las realizaciones textuales en sí, pero integrando las observaciones históricas en apariencia más heterogéneas que quedan excluidas generalmente del campo de la historia literaria” (Vaillant 2005).
} 
durante periodos significativos de tiempo, otras aparecen solo en un número de la revista que las contiene. La existencia de estas secciones y su permanencia dan cuenta del ejercicio de formación de un público lector específico por parte de las revistas. Lectores y publicaciones construyen un horizonte de expectativas para el género que cobija tanto elementos básicos asociados al género en su connotación popular (simple relato de una serie de acontecimientos) como rasgos más específicos que ponen de manifiesto la especialización del género, y al tiempo su plasticidad. La siguiente tabla presenta las secciones de cuento a las que aludimos:

\begin{tabular}{|c|c|}
\hline \multicolumn{2}{|c|}{ Tabla 1. Secciones de cuento } \\
\hline Revista & Secciones \\
\hline Sábado (Medellín, 1921-1929) & Cuento; Cuentos a María Luz; Los cuentos de "Sábado" \\
\hline $\begin{array}{c}\text { Letras y Encajes (Medellín, } \\
\text { 1926-1958) }\end{array}$ & Cuento del día \\
\hline $\begin{array}{c}\text { La Novela Semanal } \\
\text { (Bogotá/Barranquilla, 1923- } \\
\text { 1930) }\end{array}$ & El Cuento Semanal \\
\hline El Gráfico (Bogotá, 1910-1941) & $\begin{array}{l}\text { Cuento; Cuento Argentino; Cuento del Sábado; Cuento } \\
\text { Dominicano; Cuento Extranjero; Cuento } \\
\text { Mexicanoacional; Cuento Uruguayo; Cuentos; Cuentos } \\
\text { Argentinos; Cuentos de "El Gráfico"; Cuentos } \\
\text { Españoles; Cuentos Exóticos; Cuentos Extranjeros; } \\
\text { Cuentos Hindús; Cuentos Nacionales; Cuentos por } \\
\text { Tagore; Cuentos Rusos; Cuentos Uruguayos; El cuento }\end{array}$ \\
\hline Cyrano (Medellín, 1921-1923) & Cuento; Cuentos de mujeres \\
\hline $\begin{array}{c}\text { Crónica (Barranquilla, 1950- } \\
\text { 1951) }\end{array}$ & $\begin{array}{l}\text { Cuento catalán; Cuento extranjero; Cuento nacional; } \\
\text { Cuento policiaco }\end{array}$ \\
\hline Cromos (Bogotá, 1916-1950) & $\begin{array}{l}\text { Cuentistas propios; Cuento criollo; Cuento de } \\
\text { ultratumba; Cuento extranjero; Cuento nacional; } \\
\text { Cuento policial; Cuentos de Cromos; Cuentos } \\
\text { Españoles de Humor; Cuentos Españoles; Cuentos } \\
\text { Humorísticos; Cuentos morales para niños formales; } \\
\text { Cuentos pintados; Cuentos propios; Cuentos Selectos; } \\
\text { Cuentos sencillos; El cuento ameno; El cuento } \\
\text { amoroso; El cuento célebre; El cuento cómico; El } \\
\text { cuento corto; El cuento de acción; El cuento de } \\
\text { aventura; El cuento de Cromos; El cuento de guerra; El } \\
\text { cuento de intriga; El cuento de misterio; El cuento de } \\
\text { navidad; El cuento doloroso; El cuento dramático; El } \\
\text { cuento emotivo; El cuento exótico; El cuento } \\
\text { fantástico; El cuento frívolo; El cuento grotesco; El } \\
\text { cuento histórico; El cuento humano; El cuento } \\
\text { humorístico; El cuento ingenuo; El cuento irónico; El } \\
\text { cuento macabro; El cuento pasional; El cuento patético; } \\
\text { El cuento policiaco; El cuento policial; El cuento } \\
\text { psicológico; El cuento romántico; El cuento sarcástico; } \\
\text { El cuento satírico; El cuento sentimental; El cuento }\end{array}$ \\
\hline
\end{tabular}




\begin{tabular}{|c|c|} 
& $\begin{array}{c}\text { trágico; El cuento vernáculo; Los mil cuentos y un } \\
\text { cuento de Cromos }\end{array}$ \\
\hline Claridad (Medellín, 1930) & Cuentistas jóvenes \\
\hline Caminos (Barranquilla, 1922) & Cuento de primavera \\
\hline
\end{tabular}

Evidentemente, El Gráfico y Cromos son las dos publicaciones que más utilizan el recurso de la sección específica. En general, priman la procedencia del relato o una marca temática en la denominación de las secciones. La diferenciación entre la producción vernácula y la extranjera se establece como un rasgo fundamental en la caracterización de los relatos. Sería necesario profundizar en este fenómeno con la finalidad de tratar de explicar la relación que las mismas revistas construyen con la literatura nacional y la extranjera. También llama la atención el protagonismo de las temáticas o subgéneros de las narraciones a la hora de nombrar las secciones. Indudablemente el lector parte de una clave de lectura que afecta su ejercicio lector; los términos que acompañan la denominación genérica y aluden a la temática asimismo pueden funcionar como elemento atractivo para el público y animar la lectura: amoroso, cómico, de intriga, pasional, trágico.

Un aspecto fundamental, en el cual no nos detendremos, es el análisis de los relatos mismos y de los textos críticas sobre el cuento. A partir de este tipo de estudios asimismo podría rastrearse con mayor profundidad la evolución de la noción de cuento y las diferentes realizaciones del género en las páginas de las revistas. Es una tarea por hacer.

\subsection{El lugar del texto en la revista: hacia la especialización del medio}

Una característica propia de la revista es su estructura basada en secciones, sean o no fijas, tengan o no una denominación. Las decisiones del editor(es) a la hora de disponer los diferentes elementos que componen cada número afectan el sentido de los contenidos y la disposición del público lector hacia los mismos. Como bien señala Beatriz Sarlo "el tipo de letra y el lugar en las páginas de una revista pertenecieron a un conjunto de decisiones tomadas que, básicamente, son la revista misma [...] la sintaxis de una revista informa, de un modo que jamás podrían hacerlo sus textos considerados individualmente, de la problemática que definió aquél presente” (1992, p. 10). En este sentido resulta válido preguntarse por el lugar que cada publicación otorga entre sus páginas a los cuentos que publica. Así, en función del lugar que otorgan a los cuentos, nos permitimos clasificar en tres tipos las revistas analizadas y, con ello, llamar la atención acerca del valor que le otorgan al género:

a. Revistas que incluyen cuentos y géneros próximos con cierta frecuencia, aunque no cuentan con secciones específicas. Tales son los casos de La Gruta, Lectura y arte, Germinal, Revista Contemporánea, Revista Nueva, Lectura Amena, Trofeos, La Niñez, Cvltvra, Revista Moderna y Voces. Estas publicaciones utilizan la denominación "cuento" esporádicamente; la mayor parte de ellas son publicadas durante los primeros años del siglo XX. 
b. Revistas que cuentan con secciones específicas para el cuento o que constituyen plataformas para los concursos y premios. Alpha y El Gráfico marcan un punto de inflexión en el corpus estudiado debido a la importancia que otorgan al género, con ellas aparece este segundo tipo de revista. Alpha incluye un cuento en cada uno de sus números (en algunos casos publica un título en varias entregas); El Gráfico, por su parte, cuenta con las secciones "Cuentos Nacionales", "Cuentos Extranjeros", "Cuentos del Sábado" y "El Cuento". Caso similar es el de la revista Cromos, donde aparece el uso reiterado de la etiqueta "cuento" y la inclusión de secciones (ver Tabla 1); la presencia de autores colombianos es permanente, sin restar importancia a los extranjeros (destaca la presencia de franceses y españoles). Las publicaciones medellinenses Cyrano, Sábado y Letras y Encajes son plataformas destacadas para el género; Cyrano privilegia a los autores colombianos en su sección "Cuento". Sábado, por su parte, mantiene la sección "Los cuentos de Sábado", ofrece sus páginas tanto a la cuentística nacional como a la foránea. Letras y Encajes, especializada en el público femenino, cuenta con varias secciones, entre estas "El cuento" y "Página del cuento", donde divulga obra de autores naciones y extranjeros; la obra de plumas femeninas asimismo es destacable y obedece al carácter de la publicación; otro aspecto que debe subrayarse es que promueve un concurso de cuento para mujeres. La barranquillera Caminos le apuesta asimismo al género cuento al publicar entregas del mismo en cada uno de los cinco números ubicados. Revistas como Claridad, Chanchito y Rin-Rín, que aparecen con posterioridad a las antes referidas, ofrecen espacio permanente al género, en el marco de su interés por divulgar diversidad de contenidos. La revista barranquillera Crónica ofrece un espacio definitivamente protagónico al género en sus tres secciones: "Cuento extranjero", "Cuento nacional” y "Cuento policiaco”. El Gráfico, Cromos, Letras y Encajes, Revista de las Indias y Espiral, debido a sus largos periodos de circulación y cantidad de números publicados, ofrecen una perspectiva singular para el análisis del género.

c. Revistas especializadas en el género. Tal es el caso de El cuento semanal, La novela semanal y Lectura breve. Las tres colecciones de revistas, que circulan durante la década de los años veinte, evidencia una suerte de consolidación del género en el marco de un fenómeno editorial que se caracteriza por los grandes tirajes y la apuesta por la popularización del texto literario. ${ }^{6}$ La aparición de estos tres títulos en Colombia puede vincularse, además, al auge y éxito de colecciones similares editadas en Francia, España, Argentina y México. ${ }^{7}$ Indicios de que este tipo de colecciones llegan al país en esa época son la reseña de la publicación española El cuento semanal ilustrado en la revista Trofeos, las similitudes en los título y formatos de las publicaciones, la inclusión en las colecciones colombianas de obras y autores antes incluidos en las colecciones extranjeras y la existencia de algunas de estas publicaciones seriadas en bibliotecas colombianas. De las tres

6 El cuento semanal, por ejemplo, informa en el encabezado de cada entrega que su tiraje es de 5.000 ejemplares.

7 España: El cuento semanal (1907-1912), La novela corta (1916-1925), La novela de hoy (1922-1932), La novela semanal (1921-1935). Argentina: La novela de hoy (1918-1919), La novela de la juventud (19201922), La novela nacional (1920-1922), La novela para todos (1918-1919), La novela semanal (1917-1922), El cuento ilustrado (1918). Historiadores de las letras españolas proponen como modelos de las publicaciones españolas a colecciones francesas como: Lisez-moi, Les Romans de L'Illustration, La Petite Illustration (Puente, 2009 y Sarlo, 1986). 
colecciones colombianas, dos fueron iniciativa de Luis Enrique Osorio; la más exitosa de todas fue sin lugar a dudas La novela semanal que alcanzó los 125 números.

Es así como hemos podido constatar el protagonismo que van cobrando los cuentos y géneros próximos en las publicaciones: de la inserción esporádica de algún relato se pasa a la aparición constante de cuentos número a número, luego a la especialización en secciones y a la edición de títulos centrados en el género.

Otro aspecto que merece revisión es la coexistencia de elementos en las páginas de las revistas. Los conceptos de "contexto de publicación” y "contexto de edición”, como son entendidos por Annick Louis (2014), ofrecen una línea de análisis de los vínculos entre textos, ilustraciones, tipografías, disposición de elementos, coincidencia en la misma página, en la misma sección en el mismo número de la revista. A manera de ejemplo, presentamos algunas observaciones a partir de dos casos concretos:

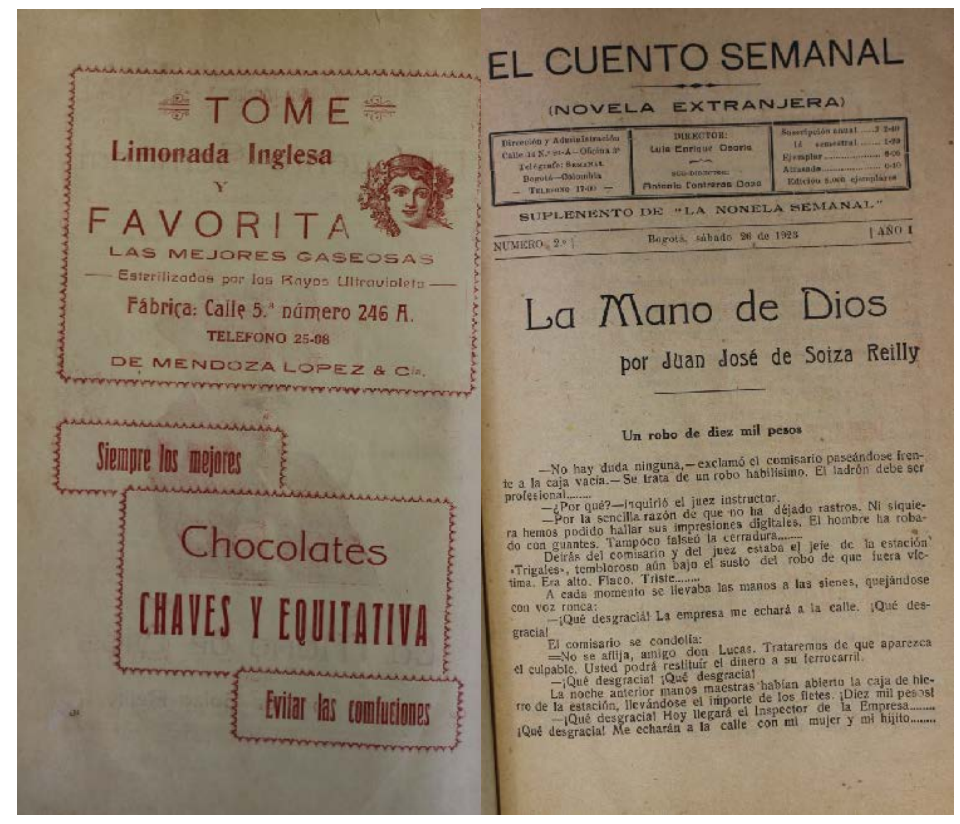

Imagen 1. Páginas 2 y 3 del número 2 de El cuento semanal

En el caso de este ejemplar de El cuento semanal, la narración es precedida por una página publicitaria — contraportada - que anuncia productos alimenticios e introduce elementos gráficos llamativos; los productos anunciados delimitan un público consumidor objetivo que coincidiría con el lector ideal propuesto por la publicación. El relato aparece en la siguiente página, comparte espacio con información relativa a la entrega (fecha, etiquetas genéricas, número y demás datos). El diseño editorial se asemeja al de un libro. El hecho de que el cuento aparezca en primer lugar y no comparta espacio con otros textos literarios denota la jerarquía de la que goza. 


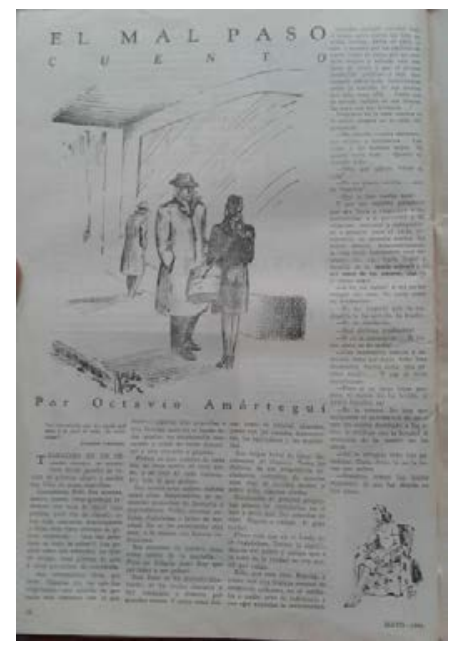

Imagen 2. Páginas 12 y 13 del número 2 de la revista Espiral

En el caso de este ejemplar de la revista Espiral, el cuento no ocupa un lugar tan destacado como en el caso anterior, está ubicado en las páginas 12-13 de la entrega, comparte espacio con una caricatura de tono político y con el fragmento final de otro artículo, de carácter crítico a propósito de la educación superior. Cabe destacar que el cuento está acompañado de ilustraciones directamente vinculadas al mismo.

Estas observaciones generales posibilitan aventurar unas primeras reflexiones acerca del tipo de lector al cual van dirigidas una y otra publicación; la articulación con otros elementos textuales y gráficos otorga diferente estatus al relato. La revisión, en todas las revistas, de aspectos como los antes nombrados es un ejercicio pendiente, que posiblemente arroje interesantes resultados.

\section{El cuento en las revistas: los datos hablan}

\subsection{Publicación de cuento en las revistas}

Nos detendremos a continuación en algunos datos que consignamos en la base de datos levantada gracias al proceso de revisión de las fuentes. Proponemos llamar la atención sobre algunos fenómenos y dejar abiertas líneas de discusión y reflexión. Partimos de señalar que hemos ubicado 6425 entradas correspondientes a títulos de cuentos y géneros próximos. El gráfico 2 ilustra la magnitud de cuentos publicados por década: 


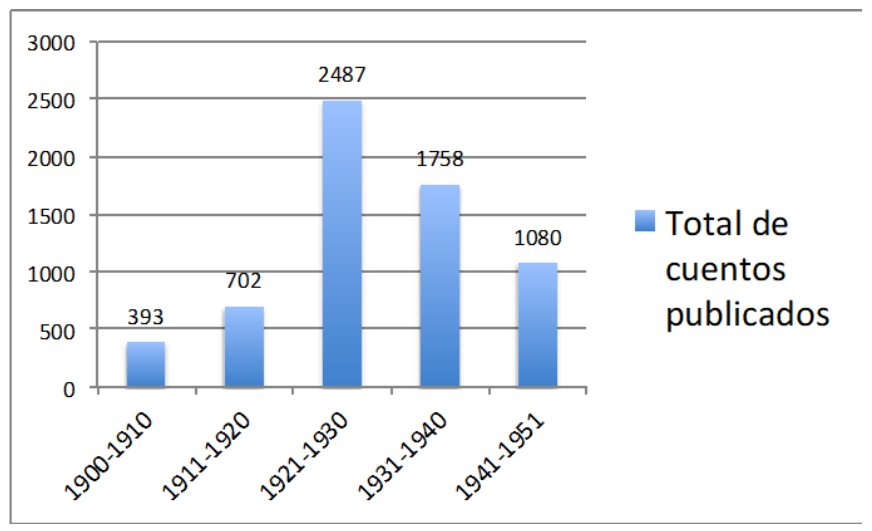

Gráfico 2. Cantidad de cuentos publicados por década

El cuento y los géneros próximos aumentaron significativamente su presencia en las revistas analizadas a lo largo del tiempo. Durante la década que va de 1921 a 1930 los relatos se triplicaron. Esta información se corresponde con las observaciones acerca de la presencia del género en la sintaxis de las revistas, con la paulatina aparición de secciones y publicaciones especializadas. Asimismo podría relacionarse con la aparición de publicaciones tan sólidas — en cuanto duraderascomo El Gráfico, Cromos y El cuento semanal. Sin embargo, y pese a que la presencia del género sigue siendo destacable, durante las décadas de los años cuarenta y cincuenta la cantidad de relatos decae. Asociamos tal fenómeno a la culminación de El Gráfico en 1941 y al cambio que sufrió Cromos a principios de la década de los cuarenta, pues ambas revistas destacan por la cantidad de cuentos que publicaron hasta $1941 .^{8}$

Algunas revistas se destacan por la cantidad de relatos publicados, lo cual encuentra una explicación, en parte, en los largos periodos de circulación de los que gozaron; sin embargo, vale la pena tener en cuenta la proporción de cuentos publicados en función del periodo de circulación de la revista para hacerse a una idea del protagonismo que cada publicación otorga a los relatos (ver tabla 2):

\begin{tabular}{|}
$\mid$\begin{tabular}{|c|c|c|c|c|}
\hline \multicolumn{5}{|c|}{ Tabla 2. Revistas, periodos de circulación y cantidad de cuentos publicados } \\
\hline Revista & $\begin{array}{c}\text { Ciudad y periodo de } \\
\text { circulación }\end{array}$ & $\begin{array}{c}\text { Años en } \\
\text { circulación/ } \\
\text { revisados }\end{array}$ & $\begin{array}{c}\text { Total de } \\
\text { cuentos }\end{array}$ & $\begin{array}{c}\text { Promedio } \\
\text { cuentos/año }\end{array}$ \\
\hline Alpha & Medellín, 1906-1912 & 6 & 150 & 25 \\
\hline Caminos & Barranquilla, 1922 & 1 & 12 & 12 \\
\hline Claridad & Medellín, 1930 & 1 & 80 & 80 \\
\hline Cromos & $\begin{array}{c}\text { Bogotá, 1916-1950 [la } \\
\text { revista aún circula] }\end{array}$ & 34 & 3966 & 116 \\
\hline Crónica & Barranquilla, 1950-1951 & 1 & 87 & 87 \\
\hline
\end{tabular}
\end{tabular}

\footnotetext{
${ }^{8}$ En el artículo escrito por Diana Barrios para el monográfico del que hace parte el presente texto se explica tal situación de Cromos.
} 


\begin{tabular}{|c|c|c|c|c|}
\hline Cvltvra & Bogotá, 1915-1920 & 5 & 23 & 4 \\
\hline Cyrano & Medellín, 1921-1923 & 2 & 185 & 92 \\
\hline $\begin{array}{l}\text { El cuento } \\
\text { semanal }\end{array}$ & Bogotá, 1923 & 1 & 26 & 26 \\
\hline El Gráfico & Bogotá, 1910-1941 & 31 & 708 & 22 \\
\hline Espiral & $\begin{array}{c}\text { Bogotá, 1944-1950 [circuló } \\
\text { hasta 1975] }\end{array}$ & 6 & 25 & 4 \\
\hline Germinal & Bogotá, 1904-1905 & 1 & 34 & 34 \\
\hline La Gruta & Bogotá, 1902-1904 & 2 & 51 & 25 \\
\hline $\begin{array}{l}\text { La Novela } \\
\text { Semanal }\end{array}$ & $\begin{array}{l}\text { Bogotá/Barranquilla, 1923- } \\
1930\end{array}$ & 7 & 100 & 14 \\
\hline Lectura Amena & Medellín, 1904-1906 & 2 & 83 & 41 \\
\hline Lectura breve & Medellín, 1923 & 1 & 26 & 26 \\
\hline Lectura y arte & Medellín, 1903-1905 & 2 & 31 & 15 \\
\hline $\begin{array}{l}\text { Letras y } \\
\text { Encajes }\end{array}$ & $\begin{array}{l}\text { Medellín, 1926-1950 [circuló } \\
\text { hasta 1958] }\end{array}$ & 24 & 131 & 5 \\
\hline $\begin{array}{c}\text { Revista } \\
\text { Contemporánea }\end{array}$ & Bogotá, 1904-1905 & 1 & 16 & 16 \\
\hline $\begin{array}{l}\text { Revista de las } \\
\quad \text { Indias }\end{array}$ & Bogotá, 1936-1951 & 15 & 65 & 4 \\
\hline $\begin{array}{l}\text { Revista } \\
\text { Moderna }\end{array}$ & Bogotá, 1915-1916 & 1 & 23 & 23 \\
\hline Revista Nueva & Manizales, 1904-1911 & 7 & 19 & 3 \\
\hline Sábado & Medellín, 1921-1929 & 8 & 545 & 60 \\
\hline Trofeos & Bogotá, 1906-1908 & 2 & 19 & 9 \\
\hline Voces & Barranquilla, 1917-1920 & 3 & 26 & 8 \\
\hline
\end{tabular}

Al observar la tabla 2 podemos recalcar no solo la labor de Cromos y El Gráfico en la difusión del cuento, también el importante papel de proyectos como Claridad, Sábado, Cyrano, Crónica, pues proporcionalmente ofrecen un espacio al género de similar magnitud al de los dos primeros títulos mencionados. Sin embrago, sería un desacierto desconocer en Cromos y El Gráfico que los largos periodos de circulación de los que gozaron muy probablemente lograron mantener un público cautivo y, por ende, un mayor impacto del género.

\subsection{El cuento: autores y procedencia}

A partir de la totalidad de títulos de cuento consignada en la base de datos, establecemos las siguientes proporciones de aparición de relatos en función de la procedencia del autor: 


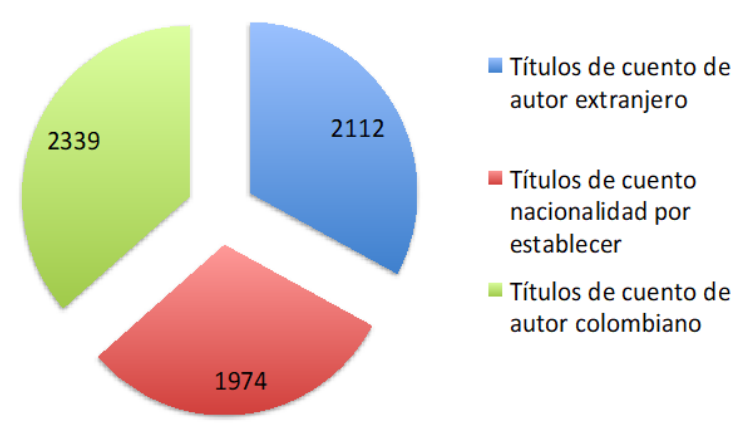

Gráfico 3. Proporción de narraciones colombianas, extranjeras y sin dato de nacionalidad

Esta imagen general, expresada en el gráfico 3, permite afirmar que el cuento de autores colombianos corre parejo al de autores extranjeros, llama la atención la abundancia de autores cuya nacionalidad no hemos podido establecer, bien sea porque las revistas no lo reportan, o porque no ha sido posible establecer el dato en otras fuentes. A lo anterior se suma la importante cantidad de relatos sin dato de autor: $749 .^{9}$

Al hilar un poco más fino y revisar en función del tiempo cómo se da la proporción de colombianos respecto a extranjeros observamos un interesante fenómeno: la presencia de autores nacionales es relativamente estable (entre $200 \mathrm{y}$ 400 entradas), sin embargo durante la década de los años veinte se presenta un aumenta sustancial del número de entradas vinculadas a autores colombianos, veamos:

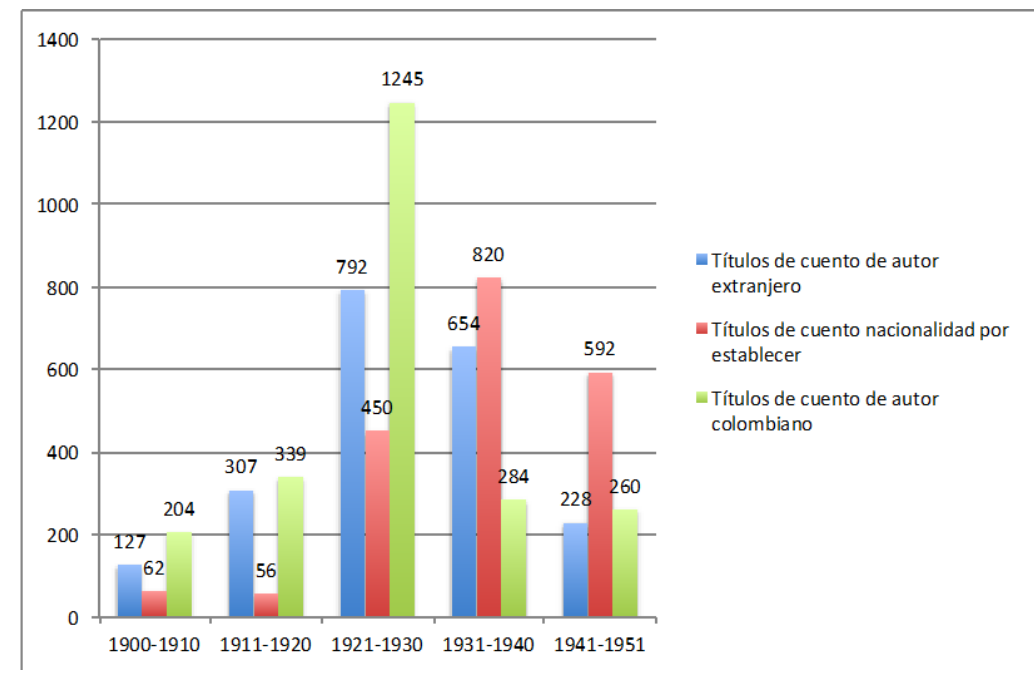

Gráfico 4. Cantidad de publicaciones colombianas, extranjeras y sin dato de nacionalidad por década

\footnotetext{
${ }^{9}$ En algunos, casos las revista no indican los nombres de los autores ni sus seudónimos (de las 6434 entradas correspondientes a cuentos o géneros próximos, 749 no cuentan con esta información, lo cual corresponde al $11,7 \%$ de las entradas, aproximadamente).
} 
La década de los años veinte se perfila como un periodo clave en el marco del corpus de revistas que hemos estudiado. La importante presencia del cuento coincide con la circulación de revistas especializadas en el género: El cuento semanal, La novela semanal, Lectura breve, asimismo con la consolidación de Cromos y El Gráfico, las cuales, pese a no ser especializadas en el género, otorgan gran protagonismo al mismo - recordemos la cantidad de secciones con que cuentan-. Es preciso emprender futuros estudios acerca de las condiciones del sistema literario colombiano durante este periodo, y en tal marco la singularidad del cuento y la prensa literaria.

Como mencionamos antes, el universo de autores establecido a partir de la revisión incluye desde nombres con una sola aparición hasta nombres de aparición constante a lo largo de amplios periodos. La publicación reiterada de un autor nos ofrece un dato importante acerca del vínculo del mismo con el género; destacamos a continuación 35 autores colombianos, quienes cuentan con 10 o más apariciones en las revistas examinadas, y que en conjunto suman 1013 entradas (de las 2339 atribuidas a autor colombiano, ver gráfico 3).

\begin{tabular}{|c|c|c|c|}
\hline Autor & Apariciones & $\begin{array}{l}\text { Periodo de } \\
\text { circulación }\end{array}$ & $\mathbf{N}^{0}$. de revistas en las que aparece \\
\hline Pedro Gómez Corena & 124 & 1916-1946 & 2 (Cromos, El Gráfico) \\
\hline Adel López Gómez & 102 & $1922-1948$ & 7 (Destaca Cromos) \\
\hline $\begin{array}{l}\text { Enrique Pardo Farelo } \\
\text { [Luis Tablanca] }\end{array}$ & 95 & $1907-1934$ & 10 (Destacan Cromos y El Gráfico) \\
\hline Eduardo Castillo & 60 & $1917-1941$ & 3 (Destaca Cromos) \\
\hline $\begin{array}{l}\text { Alberto Sánchez de } \\
\text { Iriarte }\end{array}$ & 47 & $1916-1930$ & 1 (Cromos) \\
\hline Manuel Briceño & 44 & $1920-1928$ & 1 (Cromos) \\
\hline $\begin{array}{l}\text { Lucrecio Vélez } \\
\text { [Gaspar Chaverra] }\end{array}$ & 32 & $1906-1923$ & 4 (Destaca Alpha) \\
\hline Jorge Mateus & 30 & $1921-1930$ & 2 (Destaca Cromos) \\
\hline $\begin{array}{l}\text { José Restrepo } \\
\text { Jaramillo }\end{array}$ & 29 & $1922-1945$ & 7 \\
\hline Luis Enrique Osorio & 28 & $1922-1932$ & $\begin{array}{l}3 \text { (Destacan La novela semanal, } \\
\text { Cromos) }\end{array}$ \\
\hline Tomás Carrasquilla & 28 & $1906-1930$ & $\begin{array}{l}8 \text { (Destacan Alpha, Claridad, } \\
\text { Sábado) }\end{array}$ \\
\hline Jaime Buitrago & 26 & $1928-1950$ & 1 (Cromos) \\
\hline $\begin{array}{l}\text { Alfredo Zuluaga y } \\
\text { Gutiérrez }\end{array}$ & 25 & $1921-1923$ & 1 (Cyrano) \\
\hline Roberto Montoya & 25 & $1921-1923$ & 2 (Destaca Cyrano) \\
\hline $\begin{array}{l}\text { Miguel Santiago } \\
\text { Valencia }\end{array}$ & 23 & $1916-1928$ & 1 (Cromos) \\
\hline
\end{tabular}




\begin{tabular}{|c|c|c|c|}
\hline L. Flórez Álvarez & 22 & 1923-1945 & 1 (Cromos) \\
\hline Alfonso Castro & 21 & $1905-1939$ & 8 (No destaca ninguna) \\
\hline $\begin{array}{l}\text { Antonia Cardona } \\
\text { Jaramillo }\end{array}$ & 21 & $1939-1946$ & 2 (Destaca Cromos) \\
\hline $\begin{array}{l}\text { Francisco Gómez } \\
\text { Agudelo [F. Gómez] }\end{array}$ & 20 & $1907-1930$ & 6 (Destacan Claridad y Cyrano) \\
\hline José Alejandro Navas & 20 & $1920-1929$ & 3 (Destaca Cromos) \\
\hline Samuel Velásquez & 17 & $1903-1930$ & 5 (Destacan Sábado y El Gráfico) \\
\hline María Cano & 15 & $1921-1923$ & 1 (Cyrano) \\
\hline María Castello & 15 & $1932-1941$ & 1 (Cromos) \\
\hline $\begin{array}{l}\text { Sofía Ospina de } \\
\text { Navarro }\end{array}$ & 15 & $1922-1930$ & $\begin{array}{l}4 \text { (Destacan Letras y Encajes y } \\
\text { Lectura Breve) }\end{array}$ \\
\hline $\begin{array}{l}\text { Guillermo Pérez } \\
\text { Sarmiento }\end{array}$ & 14 & $1917-1926$ & 2 (Destaca Cromos) \\
\hline Carlos Arturo Torres & 13 & $1916-1919$ & 2 (Cromos, El Gráfico) \\
\hline José Velillo & 13 & $1921-1922$ & 2 (Destaca Cyrano) \\
\hline Luis López de Mesa & 13 & $1915-1927$ & 4 \\
\hline Francisco Giraldo & 12 & $1916-1923$ & 2 (Cromos y El Gráfico) \\
\hline Luis Tejada & 12 & $1918-1929$ & 3 (Sábado, Cromos, El Gráfico) \\
\hline Carlos Delgado Nieto & 11 & $1944-1950$ & 1 (Cromos) \\
\hline $\begin{array}{l}\text { Guillermo Jaramillo } \\
\text { González }\end{array}$ & 11 & $1926-1950$ & 1 (Cromos) \\
\hline Alberto Dow Dow & 10 & $1944-1950$ & 2 (Destaca Cromos) \\
\hline Lisandro Álvarez & 10 & 1921-1930 & 2 (Claridad y Cyrano) \\
\hline $\begin{array}{l}\text { Romulado Gallego } \\
\text { [José María Castells] }\end{array}$ & 10 & 1921-1929 & 3 (Destacan Sábado y Cyrano) \\
\hline
\end{tabular}

La información expuesta en la tabla 3 nos permite avanzar en algunas reflexiones. En primer lugar, resulta destacable la cantidad de autores poco reconocidos por la historia y la crítica literarias. En segundo lugar, se evidencian dos categorías de autor en función de la cantidad de revistas a las que se vinculan: tenemos figuras como Pedro Gómez Corena, Alberto Sánchez de Iriarte, Manuel Briceño, Alfredo Zuluaga y Gutiérrez, Miguel Santiago Valencia, María Cano, entre otros, que solo aparecen en una sola publicación (o por lo menos el 90\% de sus obras); por otro lado, tenemos autores que aparece en diferentes revistas, son destacables los casos de Enrique Pardo Farelo [Luis Tablanca], Adel López Gómez, Tomás Carrasquilla, Alfonso Castro. La aparición en uno o en varios medios suscita cuestionamientos acerca de los diversos grados de legitimidad que logra un autor, en función de la diversidad de publicaciones periódicas que le ofrecen sus páginas. Un tercer aspecto que merece revisión es la relación entre el número de obras publicadas y el periodo de circulación de las mismas: por ejemplo, Manuel Briceño cuenta con 44 relatos publicados en Cromos entre 19201928 mientras que Lucrecio Vélez [Gaspar Chaverra] publicó 33 relatos en 4 
revistas entre 1906 y 1923. Estos periodos de circulación asimismo evidencian diferentes niveles de recepción y legitimación de las obras en distintos momentos del sistema literario. Sería interesante revisar qué condiciones posibilitan la "reaparición de autor" en momentos específicos o la presencia constante durante periodos cortos.

La presencia de narradores extranjeros es asimismo constante, ya lo hemos expuesto antes. Cuentos y géneros próximos de autores de diferentes países llegan a los lectores colombianos gracias a las revistas analizadas.

\begin{tabular}{|l|l|}
\hline \multicolumn{2}{|c|}{ Tabla 4. Cantidad de entradas por país } \\
\hline País & Entradas asociadas al país \\
\hline Francia & 543 \\
\hline España & 460 \\
\hline Reino Unido & 233 \\
\hline Argentina & 155 \\
\hline Estados Unidos & 135 \\
\hline Rusia & 75 \\
\hline Italia & 68 \\
\hline Uruguay & 40 \\
\hline México & 39 \\
\hline Chile & 37 \\
\hline Bélgica & 28 \\
\hline Perú & 27 \\
\hline Bangladés & 26 \\
\hline Venezuela & 26 \\
\hline Portugal & 23 \\
\hline España/Cuba & 17 \\
\hline Grecia - Irlanda & 17 \\
\hline Suecia & 17 \\
\hline Cuba & 14 \\
\hline Brasil & 13 \\
\hline Alemania & 12 \\
\hline Guatemala & 11 \\
\hline Nicaragua & 10 \\
\hline
\end{tabular}




Austria, Bolivia, Hungría, República
Dominicana, Suiza, Costa Rica,
Ecuador, Canadá, Noruega, Puerto 1 y 9 apariciones
Rico, República Libanesa, India,
Polonia, Turquía, Checoslovaquia,
Japón, Luxenburgo, Salvador,
Ucrania, Bulgaria, China, Croacia,
Dinamarca, Nueva Zelanda, Panamá,
Persia, Rumania.

Del total de entradas cuya nacionalidad fue posible establecer, tenemos la presencia de autores de 48 procedencias (aparte de la colombiana) y 7 autores de doble nacionalidad. Entre 1900 y 1950, obras francesas y españolas se destacan por su cantidad y por sobrepasar significativamente en número los relatos de otros países. Si tomamos como universo 2112 entradas asociadas a autores extranjeros, los registros franceses constituirían un 25\%, mientras que los españoles un $22 \%$. En total, $47 \%$ de los registros corren por cuenta de estos dos países europeos. Este fenómeno podría tener una explicación en las dinámicas entabladas entre los tres sistemas literarios — colombiano, español y francés - ya desde el siglo XIX. La perspectiva de las transferencias culturales ofrece valiosas herramientas de análisis de esta relación que vincula tanto las dinámicas literarias como las periodísticas; además de los vectores que facilitaron la circulación de textos extranjeros en publicaciones colombianas, sería posible estudiar los mecanismos de la traducción y las estrategias para la selección y disposición de obras originalmente escritas en otras lenguas. Es una perspectiva que enunciamos y queda abierta a futuros análisis.

Los datos escuetos que antes ofrecimos abren la puerta a análisis más detallados; así, podemos identificar los autores extranjeros más publicados en las revistas colombianas (10 o más apariciones):

\begin{tabular}{|l|l|l|}
\hline \multicolumn{2}{|c|}{$\begin{array}{l}\text { Tabla 5. Autores extranjeros con mayor presencia en revistas } \\
\text { colombianas }\end{array}$} \\
\hline Autor & $\begin{array}{l}\text { Nacionalidad del } \\
\text { autor }\end{array}$ & $\begin{array}{l}\text { eúmero de } \\
\text { entradas }\end{array}$ \\
\hline Antonio Guardiola & España & 60 \\
\hline Edgar Wallace & Reino Unido & 56 \\
\hline Georges Pourcel & Francia & 28 \\
\hline $\begin{array}{l}\text { José Augusto Trinidad Martínez } \\
\text { Ruiz [Azorín ] }\end{array}$ & España & 27 \\
\hline Enrique Banchs & Argentina & 23 \\
\hline Rabindranath Tagore & Bangladés & 23 \\
\hline E. Phillips Oppenhein & Reino Unido & 22 \\
\hline $\begin{array}{l}\text { Charles Louis Lucien [Pierre } \\
\text { Valdagne] }\end{array}$ & Francia & 19 \\
\hline Eduardo Zamacois & España & 18 \\
\hline
\end{tabular}




\begin{tabular}{|l|l|l|} 
Frederic Boutet & Francia & 18 \\
\hline Patrick Lafcadio Hearn & Grecia - Irlanda & 16 \\
\hline Alfonso Hernández Catá & España/Cuba & 15 \\
\hline Anton Chéjov & Rusia & 14 \\
\hline Guy de Maupassant & Francia & 14 \\
\hline Liev Tolstoi & Rusia & 14 \\
\hline $\begin{array}{l}\text { Oscar Fingal O'Flahertie Wills } \\
\text { Wilde [Oscar Wilde] }\end{array}$ & Reino Unido & 14 \\
\hline Catulle Mendès & Francia & 13 \\
\hline Charles-Henry Hirsch & Francia & 13 \\
\hline Henri-Georges Jeanne & Francia & 13 \\
\hline Fenimore Cooper & Estados Unidos & 12 \\
\hline Giovanni Papini & Italia & 11 \\
\hline Michel Zamacois & Francia & 11 \\
\hline Jacques Constant & Francia & 10 \\
\hline Julio Dantas & Portugal & 10 \\
\hline
\end{tabular}

No resulta extraño que la mayor parte de estos autores sea de nacionalidad francesa o española. Aparte de estos nombres, vale la pena destacar otros franceses y españoles de aparición recurrente. Entre los primeros tenemos a Marie-JosephAlbert-François Jean, Edmond Jaloux, Frederic Boutet, Henri Duvernois, Séverin Faust, Efraín Mikhael, Jacques Cesanne, Maurice Renard, Pierre Mille, Remy de Gourmont. Entre los españoles tenemos a Gregorio Martínez Sierra, Ramón del Valle Inclán, Vicente Blasco Ibáñez, Emilia Pardo Bazán, Ramón Gómez de la Serna, G. Pérez Sarmiento, Santiago Rusiñol y Prats, Jacinto Benavente, Alfonso María de Ávila, Basilia Rodríguez Espina, Joaquín Dicenta.

Cabe destacar la presencia de las narrativas, argentina, estadounidense y de Reino Unido, las de más frecuente aparición después de la española y de la francesa, aunque con una diferencia destacable en cifras. Vale la pena, en este punto, detenernos en la presencia de la cuentística del resto de Latinoamérica, la cual corresponde a 402 entradas:

\begin{tabular}{|l|l|}
\hline \multicolumn{2}{|c|}{$\begin{array}{c}\text { Tabla 6. Prencia de cuento latinoamericano en } \\
\text { revistas colombianas }\end{array}$} \\
\hline Nacionalidad del autor & Apariciones \\
\hline Argentina & 155 \\
\hline Uruguay & 40 \\
\hline México & 39 \\
\hline Chile & 37 \\
\hline Perú & 27 \\
\hline Venezuela & 26 \\
\hline Cuba & 14 \\
\hline
\end{tabular}




\begin{tabular}{|l|l|} 
Brasil & 13 \\
\hline Guatemala & 11 \\
\hline Nicaragua & 10 \\
\hline Bolivia & 7 \\
\hline República Dominicana & 6 \\
\hline Costa Rica & 5 \\
\hline Ecuador & 5 \\
\hline Puerto Rico & 4 \\
\hline Salvador & 2 \\
\hline Panamá & 1 \\
\hline
\end{tabular}

La diferencia entre la presencia en Colombia de cuentística europea y anglosajona, y la cuentística latinoamericana sugiere algunas hipótesis respecto a los vínculos entre sistemas literarios. Entre otras, que las relaciones del medio literario colombiano son más fuertes con el ámbito europeo que con las otras naciones latinoamericanas, posiblemente debido a la validación de modelos culturales del otro lado del Atlántico. Queda por estudiar con detalles, a partir de las revistas, la dinámica entre sistemas literarios latinoamericanos y el papel jugado por las nociones europeas y anglosajones en dicha dinámica. Por lo pronto, presentamos los autores más publicados del ámbito latinoamericano en las revistas estudiadas:

\begin{tabular}{|l|l|l|}
\hline \multicolumn{3}{|c|}{ Tabla 7. Autores latinoamericanos con mayor presencia en revistas colombianas } \\
\hline Autor & Nacionalidad del autor & Apariciones \\
\hline Armando Maribona & Cuba & 6 \\
\hline Enrique Banchs & Argentina & 23 \\
\hline Félix Rubén García Sarmiento [Rubén Darío] & Nicaragua & 8 \\
\hline $\begin{array}{l}\text { Juan Crisóstomo Ruiz de Nervo y Ordaz } \\
\text { [Amado Nervo] }\end{array}$ & México & \\
\hline Juan José De Soiza Reilly & Argentina & 9 \\
\hline Juana de Ibarborou & Uruguay & 6 \\
\hline Julio Franzoso & Argentina & 8 \\
\hline Leopoldo Lugones & Argentina & 6 \\
\hline Roberto Arlt & Argentina & 6 \\
\hline & & 8 \\
\hline
\end{tabular}

\section{Proyecciones de la investigación. A manera de conclusión}

El examen de las fuentes ha permitido constatar el protagonismo que alcanza el cuento, y los géneros próximos, durante la primera mitad del siglo XX. Nombres de autores y títulos de obras olvidados, hasta ahora poco revisitados por los estudios literarios y desconocidos por los lectores de nuestros días, surgen de las páginas de estos impresos. Asimismo se revela una compleja relación con sistemas literarios de otras latitudes, evidente tanto en la circulación de autores de otras 
nacionalidades en las páginas de revistas colombianas como en las características de las publicaciones mismas y en el lugar que el género va ocupando en cada época en estos impresos periódicos.

Un estudio minucioso de los cambios en la noción de cuento en Colombia puede derivarse de los datos obtenidos en esta investigación, asimismo un examen de las diferentes vertientes estéticas del género a lo largo de la primera mitad del siglo XX.

Examen aparte requieren publicaciones especializadas como El cuento semanal, La novela semanal y Lectura breve, revisión que - por cierto- implicaría un análisis de su relación con colecciones similares editadas en otros países, las cuales formaron públicos lectores singulares. Igualmente merecen estudios detenidos dos publicaciones de larga duración que ofrecieron al cuento un espacio protagónico: El Gráfico y Cromos.

Los resultados obtenidos en el proyecto de investigación del cual se deriva el presente balance abonan el camino a la preparación de ediciones de rescate y antologías de cuento colombiano, al estudio contrastivo de los rasgos estéticos de la narrativa nacional y la extranjera, asimismo al análisis de la articulación de los sistemas literarios por la vía de los géneros narrativos breves.

Para cerrar, es preciso enfatizar en la necesidad de emprender la revisión de periódicos y suplementos literarios, con el ánimo de avanzar en la ruta que hemos empezado a trazar.

\section{Referencias bibliográficas}

\section{Fuentes primarias}

\begin{tabular}{|l|l|l|l|l|}
\hline Revista & $\begin{array}{l}\text { Directores / } \\
\text { Redactores }\end{array}$ & Lugar & Periodo & $\begin{array}{l}\text { Números } \\
\text { publicados }\end{array}$ \\
\hline La Gruta & $\begin{array}{l}\text { Rafael Espinosa } \\
\text { y Federico Rivas }\end{array}$ & Bogotá & $1902-1904$ & 25 \\
\hline $\begin{array}{l}\text { Antonio J. Cano, } \\
\text { Enrique Vidal, } \\
\text { Francisco A. } \\
\text { Cano, marco } \\
\text { Tobón }\end{array}$ & Medellín & $1903-1905$ & 12 \\
\hline $\begin{array}{l}\text { Germinal } \\
\text { Julio César Arce }\end{array}$ & Bogotá & $1904-1905$ & 19 \\
\hline $\begin{array}{l}\text { Revista } \\
\text { Contemporánea }\end{array}$ & $\begin{array}{l}\text { Baldomero } \\
\text { Sanín Cano }\end{array}$ & Bogotá & $1904-1905$ & 6 \\
\hline $\begin{array}{l}\text { Revista nueva. } \\
\text { Literatura y } \\
\text { ciencias }\end{array}$ & $\begin{array}{l}\text { Rufino A. } \\
\text { Molina }\end{array}$ & Manizales & $1904-1911$ & 40 \\
\hline $\begin{array}{l}\text { Lectura amena. } \\
\text { Revista de } \\
\text { literatura }\end{array}$ & Luis Cano & Medellín & $1904-1906$ & 30 \\
\hline $\begin{array}{l}\text { Trofeos. Revista } \\
\text { de literatura } \\
\text { nacional y }\end{array}$ & $\begin{array}{l}\text { Víctor M. } \\
\text { Londoño, Ismael } \\
\text { López }\end{array}$ & Bogotá & $1906-1908$ & 15 \\
\hline
\end{tabular}




\begin{tabular}{|c|c|c|c|c|}
\hline extranjera & & & & \\
\hline Alpha & $\begin{array}{l}\text { Ricardo Olano, } \\
\text { Antonio J. Cano }\end{array}$ & Medellín & 1906-1912 & 84 \\
\hline $\begin{array}{l}\text { El Gráfico. } \\
\text { Ilustraciones, } \\
\text { información, } \\
\text { literatura y } \\
\text { variedades. }\end{array}$ & s.d. & Bogotá & $1910-1947$ & 1532 \\
\hline $\begin{array}{l}\text { Cvltvra. Revista } \\
\text { mensual }\end{array}$ & $\begin{array}{l}\text { Luis López de } \\
\text { Mesa }\end{array}$ & Bogotá & $1915-1920$ & 36 \\
\hline Revista moderna & $\begin{array}{l}\text { Arboleda y } \\
\text { Valencia }\end{array}$ & Bogotá & $1915-1916$ & 36 \\
\hline Cromos & $\begin{array}{l}\text { Propietarios } \\
\text { Arboleda y } \\
\text { Valencia }\end{array}$ & Bogotá & $1916-1950^{10}$ & 1762 \\
\hline Voces & Ramón Vinyes & Barranquilla & $1917-1920$ & 60 \\
\hline $\begin{array}{l}\text { Cyrano. Revista } \\
\text { Semanal }\end{array}$ & $\begin{array}{l}\text { Emilio Montoya } \\
\text { Gaviria }\end{array}$ & Medellín & 1921-1923 & 40 \\
\hline Sábado & $\begin{array}{l}\text { Ciro Mendía, } \\
\text { Gabriel Cano }\end{array}$ & Medellín & 1921-1929 & 150 \\
\hline $\begin{array}{l}\text { Caminos. } \\
\text { Revista } \\
\text { quincenal de } \\
\text { letras }\end{array}$ & s.d. & Barranquilla & 1922 & ¿? (150 P.) \\
\hline $\begin{array}{l}\text { El cuento } \\
\text { semanal }\end{array}$ & $\begin{array}{l}\text { Luis Enrique } \\
\text { Osorio }\end{array}$ & Bogotá & 1923 & 27 \\
\hline $\begin{array}{l}\text { La novela } \\
\text { semanal }\end{array}$ & $\begin{array}{l}\text { Luis Enrique } \\
\text { Osorio }\end{array}$ & $\begin{array}{l}\text { Bogotá, } \\
\text { Barranquilla }\end{array}$ & 1923-1930 & 125 \\
\hline Lectura breve & F. Villa López & Medellín & 1923, 1925 & 21 \\
\hline $\begin{array}{l}\text { Revista Letras y } \\
\text { Encajes }\end{array}$ & $\begin{array}{l}\text { Sofía Ospina de } \\
\text { Navarro y otras } \\
\end{array}$ & Medellín & 1926-1958 & 385 \\
\hline Claridad & $\begin{array}{l}\text { J. Yepes Morales } \\
\text { y Jorge López } \\
\text { Sanín }\end{array}$ & Medellín & 1930 & 40 \\
\hline $\begin{array}{l}\text { Revista de las } \\
\text { Indias }\end{array}$ & $\begin{array}{l}\text { Arcadio Dulcey, } \\
\text { Germán } \\
\text { Arciniegas }\end{array}$ & Bogotá & 1936-1951 & 117 \\
\hline $\begin{array}{l}\text { Espiral. Revista } \\
\text { de artes y letras }\end{array}$ & $\begin{array}{l}\text { Clemente Airó y } \\
\text { otro }\end{array}$ & Bogotá & $1944-1950^{11}$ & 135 \\
\hline Crónica & $\begin{array}{l}\text { Alfonso } \\
\text { Fuenmayor }\end{array}$ & Barranquilla & 1950-1951 & s.d [57?] \\
\hline
\end{tabular}

\section{Obras citadas}

Louis, Annick, "Las revistas literarias como objeto de estudio", en Hanno Ehrlicher y Nanette Rißler-Pipka (eds.). Almacenes de un tiempo en fuga. Revistas culturales en la modernidad hispánica. Aachen: Shaker Verlag, 2014. Disponible en:

10 Corresponde al periodo estudiando; la publicación continúa vigente.

11 Corresponde al periodo estudiando; la publicación circuló hasta 1975. 
https://www.revistas-culturales.de/es/buchseite/annick-louis-las-revistas-literariascomo-objeto-de-estudio

Pacheco, Carlos, “Criterios para una conceptualización del cuento”, en Carlos Pacheco y Luis Barrera Linares (comp.). Del cuento y sus alrededores. Caracas: Monteávila Latinoamericana, 1993, pp. 13-28.

Puente Pereda, Belén. Periodismo y discurso en El cuento semanal. Tesis doctoral, Departament de Filologia Espanyola. Barcelona: Universitat Autònoma de Barcelona, 2009. Disponible en:

http://www.tesisenred.net/bitstream/handle/10803/4897/bpp1de1.pdf?sequence=1

Sarlo, Beatriz, "Intelectuales y revistas: razones de una práctica”, Cahiers du CRICCAL, n. 9-10 (1992), pp. 9-16. Disponible en: http://www.persee.fr/doc/ameri0982-92371992num911047

Sarlo, Beatriz, "La trivialidad de la belleza. La novela semanal argentina (1917-1925)", Cuadernos Hispanoamericanos, 430 (1986), pp. 121-140. Disponible en: Biblioteca Virtual Miguel de Cervantes http://www.cervantesvirtual.com/obra/la-trivialidad-de-labelleza-la-novela-semanal-argentina-1917-1925/

Thérenty, Marie-Eve. La littérature au quotidien. Poétiques journalistiques au XIXe siècle. Paris: Éd. Le Seuil, 2007.

Vaillant, Alain, "El romanticismo y el triunfo de lo impreso", Secuencia. Revista de Historia y Ciencias Sociales, $n^{\circ}$. 62 (2005), pp. 184-194. Disponible en: http://secuencia.mora.edu.mx/index.php/Secuencia/article/view/919/1373 